\title{
Der Raum der Freiheit, der Sicherheit und des Rechts: Integrationswert für Bürger und Gesellschaft, Mitgliedstaaten und Union
}

\author{
Peter-Christian Müller-Graff*
}

Über den immateriellen Wert der europäischen Integration wie generell über Werte zu sprechen, ist gefahrstiftend abstrakt. Dies gilt umso mehr, wenn dazu kurz gefasste Impulse zu geben sind, auch wenn in diesem Beitrag eingegrenzt vor allem der sogenannte Raum der Freiheit, der Sicherheit und des Rechts ${ }^{1}$ zu würdigen ist. Eine sinnvolle Reflexion über den Integrationswert ist nicht ohne Bezugspunkt möglich, also nicht ohne zunächst die Frage zu beantworten, aus welcher Sicht der Wert zu ermitteln ist (kürzer: Wert für wen?). Wertaussagen benötigen neben einem Maßstab zuallererst einen Bezugspunkt. Generell gehaltene Richtpunkte wie beispielsweise ,für die Menschen ' oder ,für das ökonomische Optimum bleiben höchst unbestimmt. Erdet man die Frage nach dem Wert Europas in der Elementaraufgabe von Politik, also im Sinne von Gerhard Leibholz in der Sicherung des Überlebens einer sich selbst bestimmenden Population und ihres Lebensmusters, ${ }^{2}$ so liegt es im Rahmen der auswärtigen Politik der Bundesrepublik Deutschland nahe, nach dem Wert Europas für Deutschland zu fragen. Dies ermöglicht zugleich einen positivistischen Bogenschlag zum Grundgesetz. „[D]em Wohle des deutschen Volkes seine Kraft [zu] widmen, seinen Nutzen [zu] mehren und Schaden von ihm [zu] wenden, das Grundgesetz und die Gesetze des Bundes [zu] wahren und [zu] verteidigen“, 3 hat jeder Bundeskanzler bei der Amtsübernahme durch Leistung seines Amtseides zu versprechen. Zum Grundgesetz zählt auch der konditionierte Auftrag zur Mitwirkung an der Europäischen Union, die bestimmten Strukturprinzipien verpflichtet ist. ${ }^{4}$ Dies ist ein Staatsziel. ${ }^{5}$ Aus dieser Orientierung heraus soll nachfolgend eine Annäherung an die Frage nach dem immateriellen Wert der europäischen Integration in der gewünschten, selbstbestimmten Zukunft versucht werden. Hierbei kann eine genaue Abgrenzung der vorgegebenen Kategorie des ,immateriellen“ Werts zum ,materiellen “ Wert (im Sinne von preisberechenbarem Nutzen) wegen potenziell allfälliger Überschneidungen offen bleiben.

Da die positivierte europäische Integration als Konzept des frei gewählten Zusammenwirkens von Staaten wesensmäßig aber nicht auf einen einzigen Staat bezogen werden kann, sondern sich aus einer Mehrzahl von Teilnehmern definiert, empfiehlt es sich, als Maßstab für die Wertermittlung von der vereinbarten gemeinsamen Erwartung der teilnehmenden

* Prof. Dr. habil. Dr. h.c. mult. Peter-Christian Müller-Graff, Universität Heidelberg.

Der Beitrag ist eine überarbeitete Fassung des Impulsreferates des Verfassers auf dem Workshop „Der Wert Europas“" am 16. Januar 2012 im Auswärtigen Amt, Berlin.

1 Im Sinne der Art. 3 Abs. 2 Vertrag über die Europäische Union (EUV) und Art. 67-89 Vertrag über die Arbeitsweise der Europäischen Union (AEUV). Vgl. dazu Peter-Christian Müller-Graff: Der Raum der Freiheit, der Sicherheit und des Rechts in der Lissabonner Reform, in: Europarecht Beiheft 1/2009, S. 105-128.

2 So die Definition von Gerhard Leibholz in seiner Göttinger Vorlesung zu Recht und Politik (Vorlesungsaufzeichnung des Autors).

3 Art. 64 Abs. 2 i.V.m. Art. 56 Grundgesetz (GG).

4 Art. 23 Abs. 1 GG.

5 Rudolf Streinz, in: Michael Sachs (Hrsg.): Grundgesetz. Kommentar, 3. Aufl., München 2006, Art. 23, Rdz. 10. 
Staaten hinsichtlich des immateriellen Wertes auszugehen. Sie ergibt sich aus der Leitzieltrias der Europäischen Union, wie sie in Art. 3 Abs. 1 EUV niedergelegt ist: nämlich als Förderung des Friedens, ihrer Werte und des Wohlergehens ihrer Völker. Der Verwirklichung dieser Trias dienen - je auf ihre Weise - die in Art. 3 Abs. 2 bis 5 EUV aufgeführten vier operativen Hauptziele der Europäischen Union: nämlich der Raum der Freiheit, der Sicherheit und des Rechts, ${ }^{6}$ der Binnenmarkt, ${ }^{7}$ die Wirtschafts- und Währungsunion ${ }^{8}$ sowie das gemeinsame auswärtige Handeln. ${ }^{9}$ Vor diesem Hintergrund lassen sich Antworten zur Frage nach dem immateriellen Wert der Integration speziell mittels des Raums der Freiheit, der Sicherheit und des Rechts über drei Teilfragen entwickeln: nach dem vereinbarten Inhalt dieses Raums, nach dessen Beitrag zur Leitzieltrias und nach dessen Beitrag zur Bewältigung einzelner anstehender Herausforderungen.

\section{Der vereinbarte Inhalt des Raums der Freiheit, der Sicherheit und des Rechts}

Während die operativen Hauptziele des Binnenmarktes, der Wirtschafts- und Währungsunion und des gemeinsamen auswärtigen Handelns vertraute Kategorien sind, bedarf der „Raum der Freiheit, der Sicherheit und des Rechts“, der vom Vertrag von Lissabon als operatives Hauptziel erstgereiht wurde, ${ }^{10}$ der näheren Bestimmung. Dahingestellt kann hier bleiben, ob dessen Voranstellung in Art. 3 EUV im Licht von Inhalt und Systematik der Verträge sinnvoll ist im Hinblick auf seine im Vergleich zum Binnenmarktziel primärrechtlichen und darin politischen Schwächen; Stichworte hierfür sind namentlich: Teilnahme nicht aller Mitgliedstaaten an dessen Entwicklung; ${ }^{11}$ keine unmittelbar anwendbaren Vertragsvorschriften, sondern lediglich Handlungsermächtigungen der Europäischen Union; ${ }^{12}$ konzeptionelle Heterogenität. ${ }^{13}$ Sein grundsätzlicher Inhalt ergibt sich aus der vertragspositiven Umschreibung des Art. 3 Abs. 2 EUV. Dort findet sich zunächst teils zugangsversperrendes Wortgeröll. Erstens plakativ und um Wohlwollen heischend: „Die Union bietet ihren

6 Art. 3 Abs. 2 EUV i.V.m. Artt. 67ff. AEUV.

7 Art. 3 Abs. 3 EUV i.V.m. Artt. 26ff. AEUV; vgl. zur Funktion des Binnenmarkts für den Zusammenhalt der Europäischen Union André Schmidt: Die Integrationskraft des Europäischen Binnenmarkts, in: Peter-Christian Müller-Graff (Hrsg.): Der Zusammenhalt Europas - In Vielfalt geeint, Baden-Baden 2009, S. 23-42; zum Binnenmarktziel als Daueraufgabe Peter-Christian Müller-Graff: Die Verdichtung des Binnenmarktrechts zwischen Handlungsfreiheiten und Sozialgestaltung, in: Europarecht Beiheft 1/2002, S. 7-74.

8 Art. 3 Abs. 4 EUV i.V.m. Artt. 119ff. AEUV; zur integrativen Ambivalenz der Währungsunion Wim Kösters/ Tobias Zimmermann: Integrative und trennende Wirkungen der europäischen Währungsunion, in: Müller-Graff (Hrsg.): Zusammenhalt, 2009, S. 43-54.

9 Art. 3 Abs. 5 EUV i.V.m. Artt. 21ff. EUV, Artt. 205ff. AEUV; zur Funktion des auswärtigen Handelns der Union vgl. die interdisziplinäre Zusammenschau der Beiträge in Peter-Christian Müller-Graff(Hrsg.): Die Rolle der erweiterten Europäischen Union in der Welt, Baden-Baden 2006; siehe auch Alan Dashwood/Marc Maresceau (Hrsg.): Law and Practice of EU External Relation, Cambridge 2008.

10 Zuvor bezeichnete seit der Gründung der Europäischen Wirtschaftsgemeinschaft der grundlegende Aufgabenartikel die Errichtung des Gemeinsamen Marktes als erste Aufgabe (zuletzt Art. 2 Vertrag zur Gründung der Europäischen Gemeinschaft (EGV): „Aufgabe der Gemeinschaft ist es, durch Errichtung eines Gemeinsamen Marktes"). Demgegenüber fand der Raum der Freiheit, der Sicherheit und des Rechts in Art. 2 EGV keine Erwähnung und auch nur in Teilelementen in dem die Tätigkeiten ausfaltenden Art. 3 Abs. 1 lit. d EGV, sondern nur unter dem nachgereihten vierten Spiegelstrich des Art. 2 EUV der früheren Union (seit dem Vertrag von Amsterdam).

11 Dies betrifft Großbritannien, Dänemark und Irland; vgl. Protokolle Nr. 2 bis 5 zum Vertrag von Amsterdam; zu diesem Sonderweg Bernhard Kretschmer, in: Christoph Vedder/Wolff Heintschel von Heinegg (Hrsg.): Europäischer Verfassungsvertrag, Baden-Baden 2007, Art. III-267, Rdz. 14.

12 Zu dieser Delegation an die Sekundärrechtspolitik Müller-Graff: Raum der Freiheit, 2009, hier S. 112-124.

13 Dazu Müller-Graff: Raum der Freiheit, 2009, hier S. 110; Peter-Christian Müller-Graff: Der Raum der Freiheit, der Sicherheit und des Rechts - Der primärrechtliche Rahmen, in: Peter-Christian Müller-Graff (Hrsg.): Der Raum der Freiheit, der Sicherheit und des Rechts, Baden-Baden 2005, S. 11-28, hier S. 14ff. 
Bürgerinnen und Bürgern einen Raum der Freiheit, der Sicherheit und des Rechts ohne Binnengrenzen“ und zweitens abschweifend ins Kleinteilige wirkend: „in dem - in Verbindung mit geeigneten Maßnahmen in Bezug auf die Kontrollen an den Außengrenzen, das Asyl, die Einwanderung sowie die Verhütung und Bekämpfung der Kriminalität“, ehe der Satz sodann zielführend in die Aussage mündet, dass in diesem Raum „der freie Personenverkehr gewährleistet ist.“ Es geht also um die allgemeine Freizügigkeit ohne Binnengrenzkontrollen, mithin anders als im Binnenmarkt hier um die Freizügigkeit auch für nicht marktaktive Personen (soweit es solche im Licht der Rechtsprechung des Europäischen Gerichtshofes $(\mathrm{EuGH})$ zur passiven Dienstleistungsfreiheit gibt - Stichwort: Entscheidung „Luisi und Carbone“). ${ }^{14}$ Allerdings schöpft Art. 3 Abs. 2 EUV den primärrechtlichen Inhalt des Raums sprachlich nicht voll aus. Hinzu zu lesen ist Art. 67 AEUV. Dieser besagt, dass es ein Raum ist, ,in dem die Grundrechte und die verschiedenen Rechtsordnungen und -traditionen der Mitgliedstaaten geachtet werden.“ Dies bezeichnet nach Freiheit und Sicherheit die Komponente des Rechts, könnte aber missverständlich wirken. Denn nicht erst der Vertrag von Amsterdam hat mit dem Kunstbegriff des Raums der Freiheit, der Sicherheit und des Rechts den Unionsraum des Rechts erfunden. Vielmehr wurde der europäische Rechtsraum bereits mit der Gründung der Europäischen Gemeinschaften in den 50er Jahren des 20. Jahrhunderts geschaffen. Dafür steht das unverändert gültige Wort von Walter Hallstein von der Europäischen Gemeinschaft als einer Rechtsgemeinschaft. ${ }^{15}$ Dies gilt gleichermaßen für die heutige Europäische Union. Sie gründet auf verbindlichen Regeln (Primärrecht) und schafft verbindliche Regeln (Sekundärrecht), die spezifische Rechtsqualität ${ }^{16}$ haben.

Demgegenüber liefert der in der Unionsgeschichte nachgeborene Raum der Freiheit, der Sicherheit und des Rechts in seinem vertragspositiven Kern speziell für das ,Recht` letztlich nur ein Fragment, nämlich den Aspekt der Justiz, und davon nur einen spezifischen Teil, nämlich die Zusammenarbeit der mitgliedstaatlichen Justizsysteme in grenzüberschreitenden Zivil- und Strafsachen. So heterogen und fragmentiert der Raum der Freiheit, der Sicherheit und des Rechts daher auch erscheint (Personenfreizügigkeit ohne Binnengrenzkontrollen, ${ }^{17}$ Zugangspolitiken, ${ }^{18}$ Kriminalitätsbekämpfung, ${ }^{19}$ justizielle Zusammenarbeit in Zivilsachen mit grenzüberschreitendem Bezug ${ }^{20}$ ), so erlaubt er im Rahmen des Vertragsrechts doch eine konzeptionelle Rekonstruktion als Raum der Personenfreizügigkeit in Sicherheit und in justiziell aktivierbaren gemeinsamen Regeln, kürzer als befriedeter Freizügigkeitsraum. ${ }^{21}$ In dieser Pointierung klingt die Entstehung der modernen Staatlichkeit in Europa

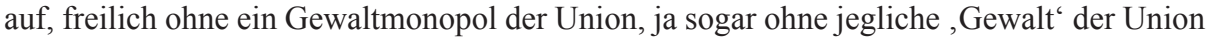
im Sinne der Verfügbarkeit legitimer physischer Gewaltmittel gegen Widerstrebende. Die

14 EuGH, Verb. Rs. 286/82 und 26/83 (Luisi und Carbone), Urteil vom 31.1.1984, Slg. 1984, 377.

15 Walter Hallstein: Die Europäische Gemeinschaft, 5. Aufl., Düsseldorf/Wien 1979, S. 51-77.

16 Der klassische Begriff des Rechts ist an die Durchsetzbarkeit durch das staatliche Gewaltmonopol gebunden. Daran partizipiert das europäische Unionsrecht über den vertraglich positivierten Grundsatz der loyalen $\mathrm{Zu}$ sammenarbeit (Art. 4 Abs. 3 EUV) in Verbindung mit den Hoheitsrechten der Europäischen Union. Eine derartige enge Verknüpfung besteht regelmäßig nicht für das klassische Völker,recht' oder das ,soft law‘.

17 Art. 67 Abs. 2, Art. 77 Abs. 1 lit. a AEUV.

18 Personenkontrollen beim Grenzübertritt an den Außengrenzen, Grenzschutzsystem an Außengrenzen, Visapolitik, Einwanderungspolitik, Asylpolitik; vgl. Art. 77 Abs. 1 lit. b und c, Abs. 2, Art. 78, Art. 79 AEUV.

19 Im Einzelnen: Justizielle Zusammenarbeit in Strafsachen (Art. 82ff. AEUV) und polizeiliche Zusammenarbeit (Art. 87ff. AEUV).

20 Art. 81 AEUV.

21 Vgl. Peter-Christian Müller-Graff: Der „Raum der Freiheit, der Sicherheit und des Rechts“ im neuen Verfassungsvertrag für Europa - Neuerungen und Notwendigkeit seiner Rekonstruktion, in: Charlotte Gaitanides/ Stefan Kadelbach/Gil Carlos Rodriguez Iglesias (Hrsg.): Europa und seine Verfassung - Festschrift zum siebzigsten Geburtstag für Manfred Zuleeg, Baden-Baden 2005, S. 605-623, hier S. 623. 
Union lebt vielmehr auch im Raum der Freiheit, der Sicherheit und des Rechts von der Loyalität der mitgliedstaatlichen Gewaltmonopole, die vertraglich auf die Unterstützung deren Aufgaben verpflichtet sind. ${ }^{22}$

\section{Der spezifische Beitrag des Raums der Freiheit, der Sicherheit und des Rechts zum immateriellen Wert der europäischen Integration}

Der spezifische Beitrag des Raums der Freiheit, der Sicherheit und des Rechts zum immateriellen Wert der europäischen Integration ist mithin zuallererst anhand der genannten vertraglich vereinbarten Leitzieltrias zu messen.

Frieden: Erstgenannt ist das Friedensziel. Inhaltlich bedeutet Frieden im Kern zuallererst die Abwesenheit von Krieg. Frieden ist gerade angesichts der trostlosen und verheerenden innereuropäischen Kriegserfahrungen über viele Jahrhunderte hinweg ein immaterieller Wert per se. Er hat zugleich eine materielle Komponente: die Vermeidung gewaltsamer Zerstörung von Vermögen. Langjähriger Frieden bietet eine Prosperitätschance, wie sie die Schweiz seit Langem erfährt.

Der Beitrag der europäischen Integration im Allgemeinen zum Frieden in der Mitte des Kontinents ist unbestritten. ${ }^{23}$ Er ist gewiss nicht der einzige Faktor. ${ }^{24}$ Aber er hat als eines der tragenden Ursprungsziele der Montanunion ${ }^{25}$ seine spezifisch pazifizierende Wirkung der Friedenssicherung durch Wirtschaftsverflechtung entfaltet. Deutschland als dem Staat in Europas Mitte mit den meisten Nachbarstaaten nutzt dies naturgemäß in besonderem Maße. Die seit dem Ende des Zweiten Weltkrieges geborenen deutschen Generationen sind seit unvordenklichen Zeiten die ersten, die bislang zeitlebens keinen Krieg im eigenen Staat erlebt haben. ${ }^{26}$ Es ist eine historische Wohltat ohnegleichen, keine kriegsbedingten Schäden an Leben, Leib, Gemüt und Vermögen erleiden zu müssen.

Unschwer lässt sich annehmen, dass zur Fortdauer dieses Zustands neben dem das Wirtschaften verflechtenden Binnenmarkt heute auch der Raum der Freiheit, der Sicherheit und des Rechts einen Beitrag leistet. Wer sich ohne Kontrolle über Staatsgrenzen bewegen kann, für den relativiert sich ein förmliches Grenzbewusstsein. Er wird zwar nicht das Andere in Regeln, Kultur und Sprache in anderen Staaten verkennen, aber dieses Andere in seinen individuellen grenzüberschreitenden Bewegungsraum als selbstverständlich aufnehmen und auf seine jeweils individuelle Weise zu verstehen versuchen. Das zukunftsträchtige Potenzial zeigt sich vielleicht am intensivsten im Erasmus-Programm: Studierende aus anderen Mitgliedstaaten sitzen in den Hörsälen beispielsweise von Heidelberg, Montpellier und Krakau, von Coimbra, Budapest und Mailand, von Prag, Cambridge und Stockholm, von Madrid, Tallinn und Brügge. Sie erleben den örtlichen akademischen Stil; sie erlernen die örtliche

22 Art. 4 Abs. 3 EUV.

23 Zur Bedeutung des Friedensgedankens bei der Entstehung der Gemeinschaften vgl. nur Jean Monnet: Erinnerungen eines Europäers, Baden-Baden 1988, S. 283-284; Hallstein: Europäische Gemeinschaft, 1979, hier S. 18; siehe auch Werner Link: Auf dem Weg zu einem neuen Europa. Herausforderungen und Antworten, Baden-Baden 2006, S. 21-30; Dieter Senghaas: Friedensprojekt Europa, Frankfurt Main 1992.

24 Stichwort zu einem weiteren maßgeblichen Faktor insbesondere: kollektives Sicherheitssystem.

25 Vgl. Monnet: Erinnerungen, 1988, hier S. 284; Hans Peter Ipsen: Europäisches Gemeinschaftsrecht, Tübingen 1972, S. 142-144.

26 Dies anerkennt trotz seines kritischen Blicks auf institutionelle Erscheinungen auch nachdrücklich hervorhebend Hans Magnus Enzensberger: Sanftes Monster Brüssel oder die Entmündigung Europas, Berlin 2011, S. 7: „Es gibt nur wenige Jahrzehnte in der Geschichte unseres Erdteils, in denen der Friede geherrscht hat. Zwischen den Staaten, die der Europäischen Union angehören, ist es seit 1945 zu keinem einzigen bewaffneten Konflikt mehr gekommen“. 
Sprache und schließen Freundschaften, vielleicht sogar Ehen; sie integrieren den Ort und dessen tradierte Kultur in ihre Biografie. Transnationale Beziehungsgeflechte entstehen. Es wäre seltsam, würde diese Erfahrung nicht Gedanken untergraben, im gemeinsamen Raum staatlich kriegerisch oder gesellschaftlich aggressiv aufeinander loszugehen.

Die Werte: Inhaltlich sind die Werte als zweiter Teil der Leitzieltrias in Art. 2 EUV im Einzelnen aufgeschlüsselt. Es ist ein hoch abstraktes Bekenntnis der Ideale der Union und der Mitgliedstaaten, denen diese laut Vertragstext gemeinsam zu sein haben. Jeder einzelne Wert ist auslegungsbedürftig und komplex. Man erkennt Inhalt und Wert der Werte bei erster Annäherung am raschesten aus ihrer Kontrastierung mit ihrer Negation: so die Achtung der Menschenwürde im Kontrast zur Objektreduzierung von Menschen ${ }^{27}$ und zur Geringschätzung des anderen Menschen; die Freiheit im Kontrast zur Fremdbestimmung der Einzelnen und des Gemeinwesens; die Demokratie im Kontrast zu einem System öffentlicher Herrschaft, das sich nicht aus dem Willen der Bürger ableitet und nicht das gleichheitssichernde Mehrheitsprinzip zur Geltung bringt; die Gleichheit im Kontrast zur ungerechtfertigten Differenzierung von Menschen; die Rechtsstaatlichkeit im Kontrast zu Regellosigkeit und öffentlicher Willkür; die Wahrung der Menschenrechte im Kontrast zur Rechtlosigkeit des Einzelnen. Hinzu kommt nach Art. 2 S. 2 EUV das Idealbild einer Gesellschaft, die sich nach den Worten der Norm ,durch Pluralismus, Nichtdiskriminierung, Toleranz, Gerechtigkeit, Solidarität und die Gleichheit von Frauen und Männern auszeichnet“. Die Werte kennzeichnen den aufgeklärt entspannten europäischen Lebensstil der dafür Empfänglichen im 21. Jahrhundert: kognitive und empathische Toleranz durch Vielfalt und für Vielfalt.

In dieser Hinsicht scheint der Beitrag der europäischen Integration zunächst schwieriger zu fassen. In den Gründungsverträgen waren die Werte nicht in der seit dem Reformvertrag von Lissabon kompakten Aufschlüsselung aufgeführt, sondern als Subtextur aufzudecken. ${ }^{28}$ Im historischen Rückblick lässt sich aber unschwer annehmen, dass nach der Überwindung des Dritten Reiches zwar nicht nur, aber gerade auch die europäische Integration die junge Bundesrepublik in ihrer organischen Werte-Entwicklung durch die Einbettung in den transnationalen Verbund derart gleichgesinnter staatlicher Gemeinwesen förderte. ${ }^{29} \mathrm{Da}$ zugleich angenommen werden kann, dass die Verwirklichung eigener Wertvorstellungen zu einem gewissen Grad auch von einer gleich gesinnten Praxis der Nachbarschaft begünstigt werden beziehungsweise von anders gesinnter Nachbarschaft gefährdet sein kann, ${ }^{30}$ vermag die europäische Integration der wechselseitigen Stabilisierung solcher Werteorientierung zu dienen - umso mehr, je substanziierter sie sich entfaltet. Eine Großleistung von historischer Dimension bewirkten diese Richtpunkte der Integration für die Wiedervereinigung Deutschlands (die Nachbarn konnten sich auf den Wertegleichklang der Bundesrepublik verlas-

27 Vgl. zur Unvereinbarkeit derartiger Objektreduktion zur Achtung der Menschenwürde im Grundgesetz zum Beispiel Bundesverfassungsgericht, Urteil vom 15.12.1970, Slg. 30, 1 (26); Bundesverfassungsgericht, Urteil vom 17.1.1979, Slg. 50, 166 (175).

28 Dies galt insbesondere für den Grundrechtsschutz, der vom EuGH unter dem Gesichtspunkt der allgemeinen Rechtsgrundsätze entwickelt wurde; grundlegend EuGH, Rs. 29/69 (Stauder/Ulm), Urteil vom 12.11.1969, Slg. 1969, 419.

29 Die Frage dieses Zusammenhangs substantiiert zu klären, ist ein Petitum an die zeitgeschichtliche Forschung.

30 So etwa bis 1989 durch die Sowjetunion und heute im globalen Kontakt durch andersartige Lebensmuster anderwärts. 
sen $)^{31}$ und bei der Transformation der ostmitteleuropäischen (beziehungsweise mittelosteuropäischen) Staaten nach der Epochenwende von 1989. ${ }^{32}$ Sie dienen heute Teilen der Eliten in östlichen und südöstlichen Nachbarstaaten als Bezugspunkt (zum Beispiel unter westwärts gewandten Akademikern in der Ukraine ${ }^{33}$ und in der Türkei $\left.{ }^{34}\right)$.

Auch für die Förderung dieser Werte durch die europäische Integration liegt der Beitrag des Raums der Freiheit, der Sicherheit und des Rechts auf der Hand. Der sichere Bewegungsraum erleichtert Zusammenkommen und Austausch. Ein Beispiel aus der Alltagspraxis mag dies illustrieren. Im deutschen Generalkonsulat in Krakau wurde im Januar 2012 bereits der 15. Jahrgang der Schule des Deutschen Rechts der Jagiellonen-Universität eröffnet. Ganz selbstverständlich, zwanglos und osmotisch sprechen dort seit Jahren polnische und deutsche Wissenschaftler und Studierende wertend und vergleichend über viele Einzelthemen der Entwicklungen der beiden Staaten. Im hermeneutischen Dialog mit Sachfragen entwickeln sich verschiedene Facetten und Verständnisse, aber auch gemeinsamer Verständnisgrund der gemeinsamen Werte.

Das Wohlergehen der Völker: Art. 3 Abs. 1 EUV spricht drittens auch allgemein das Wohlergehen der Völker an: der Völker, nicht also nur das Wohlergehen eines einzelnen Volkes. Dies beinhaltet einen staatenübergreifenden Gemeinwohlhorizont. Wohlergehen ist einerseits wirtschaftliche Prosperität, reicht aber andererseits weit darüber hinaus. Dazu rechnen auch namentlich die immateriellen Werte des sozialen Friedens, der inneren Sicherheit und des Schutzes vor äußerer Bedrohung.

Der Beitrag des Binnenmarktes zur wirtschaftlichen und sozialen Prosperität in Deutschland ist communis opinio. ${ }^{35}$ Die justizielle Durchsetzung seiner Regeln und der Rechtsschutz durch den Gerichtshof der Europäischen Union ${ }^{36}$ leisten das Ihrige. Der Beitrag der Währungsunion zum Wohlergehen wird derzeit breitflächig zu bilanzieren versucht. ${ }^{37}$ Ein freier Bewegungsraum ohne Binnengrenzkontrollen für jedermann - befriedet, gesichert, justiziell verlässlich - vermag zweifelsohne zur Steigerung des allgemeinen Wohlbefindens beizutragen. Er ist eine Errungenschaft der Integration, für die praktisch und symbolisch Schengen steht. $^{38}$

31 Vgl. Peter-Christian Müller-Graff: Deutsche Einheit und Europäische Integration, in: Beate Kohler-Koch (Hrsg.): Die Osterweiterung der EG: Die Einbeziehung der ehemaligen DDR in die Gemeinschaft, Baden-Baden 1991, S. 23-56, hier S. 41-45; zur gewollten Verzahnung von Wiedervereinigungspolitik und Europapolitik Konrad Adenauer: Erinnerungen 1955-1959, Stuttgart 1967, S. 252-255.

32 Dies manifestierte sich insbesondere in der Heranführung der ostmitteleuropäischen Staaten an die Rechtsordnung der Europäischen Union bis hinein in das Verfahren der „Kapitelbehandlung“ in den Beitrittsverhandlungen; vgl. dazu Peter-Christian Müller-Graff: Die rechtliche Dimension der Osterweiterung der Europäischen Union, in: Werner Heusel (Hrsg.): Die Osterweiterung der Europäischen Union, Köln 2002, S. 21-48.

33 Vgl. dazu namentlich Roman Petrov: Exporting the Acquis Communautaire through European Union Agreements, Baden-Baden 2011.

34 Vgl. dazu namentlich Haluk Kaabalioğlu: Turkey’s Relations with the European Union: Customs Union and Accession Negotiations, in: Peter-Christian Müller-Graff/Haluk Kabaalioğlu (Hrsg): Turkey and the European Union, Baden-Baden 2012, S. 11-27.

35 So wiesen etwa die Statistik der OECD und der Deutschen Bundesbank für den deutschen Warenexport 2005 einen Exportanteil von 63,4 Prozent in die seinerzeitige ,EU-25' auf; vgl. OECD: Monthly Statistics of Foreign Trade (FTMS), Paris 2005; Fritz Breuss: Deutschlands wirtschaftliche Stellung in der EU und in der Welt. Ein Vergleich mit Österreich, in: Peter-Christian Müller-Graff (Hrsg.): Deutschlands Rolle in der Europäischen Union, 2. Aufl., Baden-Baden 2009, S. 53-140, hier S. 86.

36 Vgl. zu dieser Grundaufgabe der Unionsgerichtsbarkeit: Art. 19 EUV, Artt. 251ff. AEUV.

37 Siehe dazu Christian Dreger: Der ökonomische Wert der Währungsunion: eine positive Bilanz aus deutscher Sicht, in diesem Band, S. 111-116.

38 Hans Claudius Taschner: Schengen. Die Übereinkommen zum Abbau der Personenkontrollen an den Binnengrenzen von EU-Staaten, Baden-Baden 1997. 


\section{Der Beitrag des Raums der Freiheit, der Sicherheit und des Rechts zur Bewältigung einzelner anstehender Herausforderungen}

Der Beitrag des Raums der Freiheit, der Sicherheit und des Rechts zur Bewältigung einzelner anstehender Herausforderungen im Sinne des Überlebens einer sich selbst bestimmenden Population und ihres Lebensmusters und darin zum immateriellen und materiellen Wert der europäischen Integration kann sich aus dem Gemeinwohlhorizont jedes Mitgliedstaats (und der Union insgesamt) jeweils spezifisch darstellen. Montesquieu hat die Bedeutung der Geografie für den jeweiligen Geist des Rechts und darin der Politik hervorgehoben. ${ }^{39}$ Speziell für Deutschland als dem Staat der zentralen Mittellage in der Union und deren zugleich nachbar- und bevölkerungsreichster Mitgliedstaat ist allerdings davon auszugehen und zu berücksichtigen, dass dessen Gemeinwohlhorizont mit dem wohlverstandenen Gemeinwohlhorizont und Erfolg der Union insgesamt eine große Schnittmenge aufweist. Gerade deshalb ist der grundgesetzliche Auftrag zur Mitwirkung an der Entwicklung der Eu-

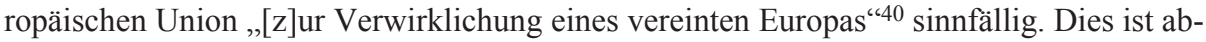
schließend im Blick auf das Potenzial speziell des allgemeinen Freizügigkeitsraums für anstehende Herausforderungen von Demografie, Kultur, Sprache und Zivilverbindungen zu betrachten.

Demografie: Es ist Gemeinwissen, dass Deutschland (aber auch eine Reihe anderer Mitgliedstaaten) ein demografisches Langfristproblem hat. ${ }^{41}$ Der amerikanische Diplomat Tony Corn hat dies jüngst unter dem Aspekt „neuer deutscher Illusionen“ essayartig pointiert. ${ }^{42}$ Zuzugeben ist ihm, dass außenpolitischer (Mit-)Gestaltungswille vor dem Hintergrund einer abnehmenden Bevölkerungszahl im Vergleich zu 1,3 Milliarden Chinesen erstaunlich wirkt - jedenfalls auf den ersten Blick. Als Hypothese mag auch hingehen, dass größere Populationen auf Dauer weniger gefährdet sind als kleinere. Corn sagt: „Das Entscheidende ist nicht die Ökonomie. Es ist die Demographie. “" ${ }^{\text {33 }}$ Schafft Nachwuchs wie Frankreich, ruft er über den Atlantik. Darin liegt freilich auch der Blick, der allein klassisch national segmentiert denkt. Er vernachlässigt die Chancen der Union. Denn Nachwuchs in ein attraktives Lebensmuster sachbezogener Tüchtigkeit und tolerant abgewogener Pluralität zu generieren, kann zwar nicht allein, aber auch durch einen Zuzugssog erfolgen.

$\mathrm{Zu}$ derartiger Mobilität innerhalb Europas bieten die integrationsrechtlich und integrationspolitisch fundamentalen Grundfreiheiten des Binnenmarkts, ${ }^{44}$ die Rechte der Unionsbürgerschaft ${ }^{45}$ und eben auch die Möglichkeiten des allgemeinen Freizügigkeitsraums eine laufende Chance. Man sollte sie überlegt nutzen, nicht aber verschenken. Derartige Mobilität vermag zugleich, grenzüberschreitende Identität in Europa und den europäischen Lebensstil zu festigen. Eine klug gehandhabte Einwanderungspolitik in Verbindung mit einer effektiven mitgliedstaatlichen Eingliederungspolitik kann einen zukunftsförderlichen Zuzug von außerhalb in die Europäische Union ermöglichen.

39 Montesquieu: L'Esprit des Lois, ediert in Tome 2 des Oeuvres Complètes de la Pléiade von Roger Caillois, Paris 1951.

40 Art. 23 GG.

41 Vgl. dazu Josef Schmid: Das demographische Profil Deutschlands und seine Stellung innerhalb der Europäischen Union, in: Müller-Graff: Deutschlands Rolle, 2009, S. 149-164.

42 Tony Corn: Neue deutsche Illusionen, in: Frankfurter Allgemeine Zeitung, 2.1.2012.

43 Ebenda.

44 Art. 28ff. AEUV.

45 Art. 20 AEUV; dazu Christoph Schönberger: Stiftet die Unionsbürgerschaft europäische Identität?, in: MüllerGraff: Zusammenhalt, 2009, S. 55-72. 
Kultur und Sprache: In Kultur und Sprache sehen nicht wenige Deutschland (ebenso wie zahlreiche andere Länder) von globalen Entwicklungen gefordert. Kultur und Sprache transportieren Denkmuster, Verständnisse, Konzepte und Lebensmuster. ${ }^{46}$ Sprache vermittelt auch Empathie und bildet professionelle Beratungsmärkte. Es hat den Anschein, als seien eigenständige Profile, Lebensmuster und Vielfalt in Bildung, Kultur, Sozialverhalten und Sprache derzeit durch universal uniformierende Auswirkungen der angelsächsischen Welt gefährdet. Europas Potenzial und Eigenheit wird damit verfehlt. Die vertraglich gewollte Chance der Union ist gerade nicht eine hegemonialartige Überformung der Kulturen und Sprachen, sondern die Förderung von Vielfalt und Pluralität. ${ }^{47}$ Die Europäische Union ist auch primärrechtlich vielsprachig in Kultur und Recht angelegt und ausgerichtet. ${ }^{48}$ Jan Sokol erkennt gerade in der Vielsprachigkeit ein Signum von Europas Identität ${ }^{49}$ und Umberto Eco lehrt, dass Babel keine Last, sondern ein Vorzug ist. ${ }^{50}$ Dies gilt gerade auch für die transnationale Kommunikation. Versuche, in Europa uniformierend eine einzige transnationale Sprache durchzusetzen, verfehlen Europas Charakter. In der Binnengrenzüberschreitung ist die Bereitschaft zu Vielseitigkeit und Offenheit gefordert.

Der freiheitliche Binnenraum der Europäischen Union bietet für dessen größte Kulturund Sprachgemeinschaft mit ihrer Mittellage zwischen West und Ost, Nord und Süd unausgeschöpfte Chancen der Kooperation. Europas immaterieller Mehrwert besteht gerade auch in der Vermeidung kultureller Einseitigkeiten und in der Förderung des für andere Kulturen offenen und achtungsbereiten Bürgers, ohne dessen Selbstbestimmung zu schädigen.

Zivilverbindungen: Die europäische Integration sieht sich nicht selten der Kritik ausgesetzt, eine bürgerenthobene, postdemokratische Veranstaltung von Eliten zu sein. So äußerte erst jüngst Hans Magnus Enzensberger provokant die These von der politischen Entmündigung der Bürger durch die Selbstreferenz der Institutionen Europas. ${ }^{51}$ Das darin angesprochene Problem der transnational gemäßen und transnational möglichen demokratischen Legitimation ist ein viel behandeltes Thema, das soeben von Jürgen Habermas mit der Akzentuierung „Entscheidung zwischen transnationaler Demokratie und postdemokratischem Exekutivföderalismus“ wieder abstrakt pointiert wurde. ${ }^{52}$ Die Frage an staatenüberlagernde Machtorganisationsmodelle im europäischen oder globalen Maßstab ist freilich neben dem Petitum der Sicherung effektiver und lebendiger Demokratie ${ }^{53}$ durch föderale Gewaltenteilung auch, ob transnationale Demokratie ohne eine hinreichend verdichtete und sich innerlich vertrauende transnationale Zivilgesellschaft überhaupt auf festem Boden gründen kann. In diese Richtung hat das Konzept der Europäischen Gemeinschaft pionierartig Möglichkeiten eröffnet: mittels Gewährung der grenzübergreifenden Marktgrundfreiheiten, also subjektiver

46 Keith Chen vertritt sogar die These von einem Zusammenhang zwischen dem Grad des Zukunftsbezugs einer Sprache (zum Beispiel höher in Deutsch, Finnisch und Koreanisch als in Griechisch, Italienisch oder Englisch) und dem wirtschaftlichen Verhalten, zum Beispiel der Höhe der Sparbereitschaft; vgl. Keith Chen: The Effect of Language on Economic Behavior: Evidence from Saving Rates, Health Behaviors, and Retirement Assets, Cowles Foundation Discussion Paper No. 1820, Yale University, New Haven, August 2011.

47 Art. 167 AEUV.

48 Art. 55 EUV.

49 Jan Sokol: Die „verschwejkte“ Reform. Ein Gespräch über Bologna, die Universität und Europa, in: Forschung und Lehre 2011, S. 428-429, hier S. 429.

50 Umberto Eco: Die Suche nach der vollkommenen Sprache, München 1994, S. 342-349.

51 Enzensberger: Sanftes Monster Brüssel, 2011, S. 50-53.

52 Jürgen Habermas: Zur Verfassung Europas, Berlin 2011, S. 48-82.

53 So insbesondere das Grundanliegen des Bundesverfassungsgerichts in seinem Lissabon-Urteil und erneut unter dem Gesichtspunkt der haushaltspolitischen Selbstgestaltungsfähigkeit im Budgethilfen-Urteil. Vgl. Bundesverfassungsgericht, Urteil vom 30.6.2009, Slg. 123, 267 und Bundesverfassungsgericht 2BvR 987/10, 2BvR 1485/10, 2 BvR 1099/10. 
Rechte der transnationalen Privatinitiative, sind Perspektiven zur Bildung einer europäischen Privatrechtsgesellschaft ${ }^{54}$ aufgetan und mittels der Schaffung der Unionsbürgerschaft verstärkt. Bemerkenswerterweise registriert auch Enzensberger die Entwicklung, wenn er befindet, an dem ,unscheinbaren Gegenstand“ von Adressbüchern lasse „sich der wahre Stand der europäischen Integration ablesen“.55 ein Gewimmel ,in diesen Notizbüchern, über ganz Europa verstreut, von [...] Geschäftspartnern, Enkeln, Kontonummern, Lehrern und Schülern, Websites, Münzsammlern, Winzern, Putzfrauen, Automechanikern, Zahnärzten und Schwarzarbeitern“. Und er schlussfolgert ,[h] $[$ eute verbinden uns die zivilen Netze stärker als alle Abkommen [...] Millionen von Fäden schaffen Interdependenzen“. ${ }^{56}$

Es liegt auf der Hand, dass das Konzept und die normative Verwirklichung des Raums der Freiheit, der Sicherheit und des Rechts solche sich von selbst vollziehende transnationale soziale Vernetzung zu begünstigen geeignet ist. In welchem Dichtegrad dies tatsächlich geschieht (für wie viele Personen welcher regionaler Herkunft und welchen Bildungsgrads in welcher Form und mit welcher Nachhaltigkeit und gegebenenfalls mit welchem Bürgersinn beziehungsweise mit welcher kommunitaristischer ${ }^{57}$ Verantwortungsbildung für eine grenzübergreifende Gemeinschaft?), ist genauerer empirischer Untersuchung wert. Je nach den zugrunde gelegten Kriterien kann daraus substanziierter als aus einer bloßen Oberflächenbeobachtung die Frage beantwortet werden, ob und gegebenenfalls in welchem Umfang und vor allem mit welchen Profilmerkmalen und in welcher Intensität sich eine staatenübergreifende europäische Gesellschaft identifizieren lässt oder nicht. Bemerkenswert offen ist der Bezugspunkt der „Gesellschaft“ im Werte-Artikel des Unionsvertrages: „Diese Werte sind allen Mitgliedstaaten in einer Gesellschaft gemeinsam“. ${ }^{58}$ Von ihm lässt sich zweierlei umfasst sehen: Gesellschaft des jeweiligen Mitgliedstaats und transnationale Gesellschaft.

Der Grad sozialer transnationaler Vernetzung kann allerdings nicht aus sich selbst heraus Antwort auf die davon zu trennende normative und darin politisch zu entscheidende Frage der zu einer derartigen transnationalen gesellschaftlichen Vernetzung gemäßen Organisation von Herrschaft im Sinne einer politischen Gemeinschaft geben. Dies gilt insbesondere für die von Habermas auf hoher Abstraktionsebene verfolgte Frage nach einem veränderten Legitimationskonzept von Hoheitsrechten in Europa und im Gefolge von Europas möglicher Schrittmacherschaft für die Welt ${ }^{59}$ (namentlich: Teilung der Legitimationsgrundlage der Hoheitsrechte der Union zwischen den staatlich organisierten Völkern und den Individuen als Unionsbürger ${ }^{60}$ oder eine gänzliche Verschiebung der Legitimationsgrundlage zu den

54 Dazu Peter-Christian Müller-Graff: Die Europäische Privatrechtsgesellschaft in der Verfassung der Europäischen Union, in: Peter-Christian Müller-Graff/Herbert Roth (Hrsg.): Recht und Rechtswissenschaft, Signaturen und Herausforderungen zum Jahrtausendbeginn. Ringvorlesung der Juristischen Fakultät der RuprechtKarls-Universität Heidelberg, Heidelberg 2000, S. 271-305; Peter-Christian Müller-Graff: Europäische Verfassungsrechtspolitik für Wirtschaft und Union: Europäische Privatrechtsgesellschaft und überstaatliche Autorität, in: Peter-Christian Müller-Graff (Hrsg.): Perspektiven des Rechts der Europäischen Union, Heidelberg 1998, S. 183-222, hier S. 195-197.

55 Enzensberger: Sanftes Monster Brüssel, 2011, S. 67.

56 Ebenda.

$57 \mathrm{Zu}$ diesem Konzept und seinen Varianten Winfried Brugger: Liberalismus, Pluralismus, Kommunitarismus. Studien zur Legitimation des Gundgesetzes, Baden-Baden 1999, S. 253-269; zum soziologischen Ansatz Philip Selznick: The Moral Commonwealth - Social Theory and the Promise of Community, Berkeley 1992.

58 Art. 2 S. 2 EUV.

59 Habermas: Verfassung Europas, 2011, hier S. 82-96: „Von der internationalen zur kosmopolitischen Gemeinschaft“ mit berechtigten „kommunitaristischen Zweifel(n) an der möglichen Transnationalität der Volkssouveränität".

60 Zu diesem Gedanken Habermas: Verfassung Europas, 2011, hier S. 62-69: „die Teilung der konstituierenden Gewalt zwischen Unionsbürgern und europäischen Völkern“. 
Individuen mit deren legitimationsspendender Doppelrolle für Staat und Union ${ }^{61}$ - Letzteres beinhaltet freilich die ungelöste zentrale Folgefrage nach der Prioritätensetzung der Legitimationsspender im Blick auf die unterschiedlichen Gemeinwohlhorizonte der beiden ,Kollektive'). Es ist auch ein anderes, an dieser Stelle nicht zur Erörterung aufgegebenes Thema, inwieweit eine mit Veränderungen im Konzept der primären Legitimation kollektiver Macht verbundene transnationale Machtaggregation ein politischer Gewinn wäre im Vergleich zu den relativ überschaubaren, demokratischen und machtteilenden Republiken der in der Europäischen Union kulturell und politisch in sich relativ homogenen und kohäsiven Gesellschaften. Der Raum der Freiheit, der Sicherheit und des Rechts erleichtert lediglich, aber immerhin das grenzüberschreitende Zusammenkommen und das Entstehen der ,Millionen von Fäden“.

\section{Chancen für Selbstbestimmung und transnationale soziale Interdependenzen}

Pointiert lässt sich aus den vorgenannten Überlegungen ein immaterieller Integrationswert mithin vor allem darin sehen, dass zum einen in paradox wirkender Weise mittels wechselseitiger Relativierung isolierter einzelstaatlicher Gemeinwohlhorizonte die Chancen für die Selbstbestimmung des jeweils eigenen Lebensmusters innereuropäisch und global gefördert und zum anderen speziell mit Blick auf den Raum der Freiheit, der Sicherheit und des Rechts die sozialen Interdependenzen der Einzelnen untereinander grenzüberschreitend ausgeweitet werden können. Die gewachsene europäische Integration bietet hierzu längst bewährte Möglichkeiten und den stabilen Rahmen zur Erprobung neuer Ansätze.

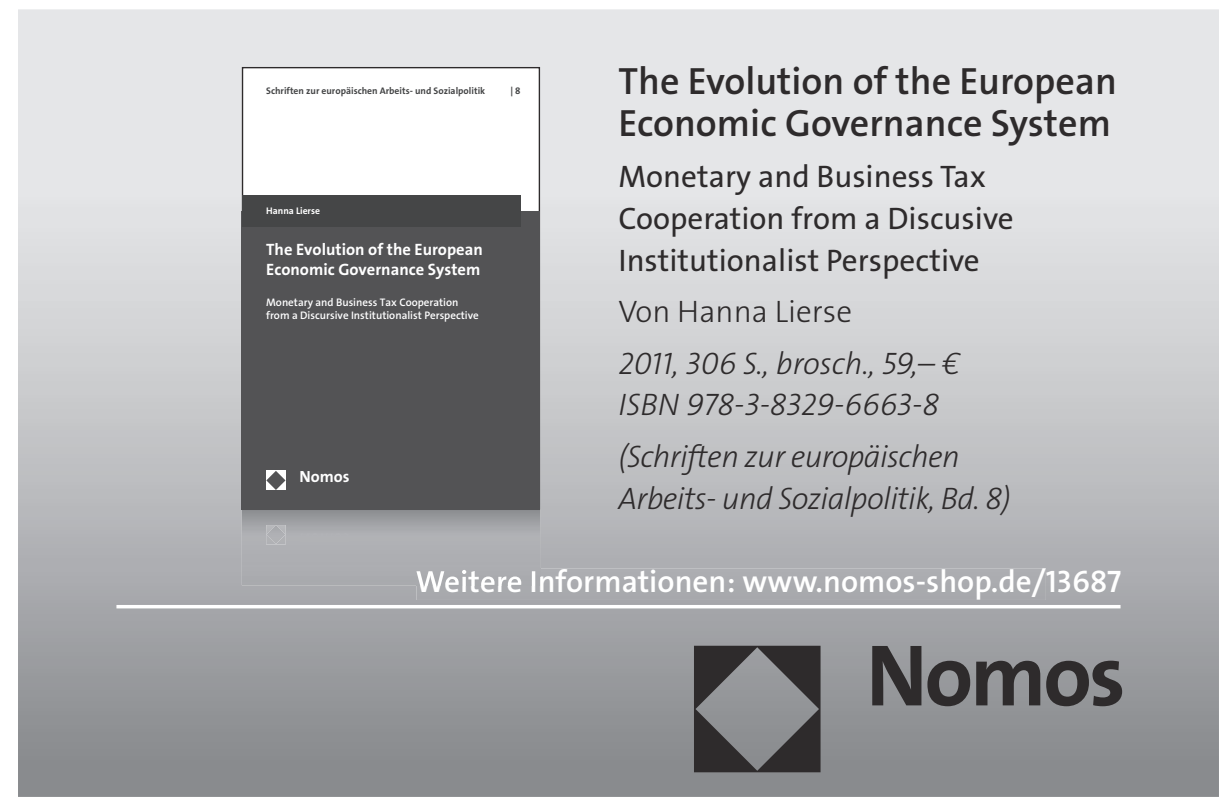

61 Habermas: Verfassung Europas, 2011, hier S. 69. 